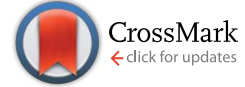

Cite this: RSC Adv., 2017, 7, 11049

Received 28th December 2016 Accepted 27th January 2017

DOI: 10.1039/c6ra28773e

rsc.li/rsc-advances

\title{
A polarized liquid metal worm squeezing across a localized irregular gap $\uparrow$
}

\author{
You-you Yao and Jing Liu*ab
}

The deformation capability of a robot has been a long-term research interest in robotic fields along with developing its controllability and adaptability. So far, many different structural designs and materials had been created to build a flexible and compliant robot. Here, we demonstrate a series of highly deformable locomotions overcoming sharp corner or small gap via EGaln liquid metal materials, which we dubbed "liquid metal worm." The worm can be controlled by exterior voltages and can veer in a loop channel. Besides, it can narrow and fit through a highly confined gap five times thinner than its own size and return to its original shape and movability. The size of the worm can also be adjusted with different volumes of liquid metal, which varies from $\sim 0.1 \mathrm{~mL}$ to $\sim 1 \mathrm{~mL}$. In addition, other solid functional structures or modified particles can be mounted according to previous research. Several phenomena regarding reconfigurational reversing of the moving direction due to metal droplet morphology were disclosed with the interpreted mechanism. The worm realized a biomimetic deformative characteristic and may have profound implications in other fields such as vascular robots and microfluidics. It is expected that the present work could contribute to the development of soft robots and their miniaturization in the coming future.

\section{Introduction}

Soft robots are a hot spot for researchers in robotic areas because of their compliant, adaptable and flexible characteristics. For biomedical applications, a soft micro-robot performs an important and promising role because of its non-invasive features. It can be utilized in multiple tasks like drug delivery, attachable wearable sensors, dredging of clogged vessels and others. Lots of technologies have been developed to design a controllable and deformable small robot driven by electricity, magnetic fields, pneumatic actuators or UV light. ${ }^{1-14}$ Many efforts in the past few years have been devoted to achieve a biomedical robot that can travel through human tubular tissues such as vessels, bronchus and intestinal tracts. ${ }^{15}$ When looking into the natural world, there are many creatures that may inspire scientists for appropriate approaches. Snakes can move ahead and swerve by reciprocating twists, ${ }^{\mathbf{1 6}, 17}$ while earthworms can have peristalsis through periodically contracting and swelling different segments of its body both spatially and temporally. ${ }^{18-20}$ The amoeba is also an attractive research target that can transform and move almost freely with its

${ }^{a}$ Department of Biomedical Engineering, School of Medicine, Tsinghua University, Beijing 100084, PR China. E-mail: yaoyy14@mails.tsinghua.edu.cn; jliubme@mail. tsinghua.edu.cn; Tel: +8601062794896

${ }^{b}$ Technical Institute of Physics and Chemistry, Chinese Academy of Sciences, Beijing 100190, PR China

$\uparrow$ Electronic supplementary information (ESI) available: More supplementary movies of the liquid metal worm. See DOI: 10.1039/c6ra28773e alterable skin, which can switch from the gelatinous state to the aqueous state. ${ }^{21}$ All these creatures can go through a hole that is relatively small compared to their body size.

In this paper, we proposed a different type of soft robot that can narrow to fit through an extremely small slit and return to its original shape based on EGaIn liquid metal material. We dubbed it a "liquid metal worm." With appropriate exterior electric stimuli, the liquid metal worm can waddle forward within a $3 \mathrm{D}$ printed channel that is filled with electrolyte solution. It can also veer in crooked channels with a long body. An interpretation of the crawling phenomenon was carried out based on observation and inspection. Basically, the cathode oriented locomotion is caused by gallium oxide layer generation and skin flow according to the Marangoni effect. Several conceptual experiments were conducted to prove the rationality of our assumption. Such a soft liquid robot may help researchers in the field of soft robots to build more flexible and adaptable equipment. ${ }^{19}$ The discovery can also influence the related vicinity research fields such nano/micro machines and micro fluidic technology, where researchers confront similar "crossing" challenges. ${ }^{22-25}$

\section{Experimental}

\section{Liquid metal material}

EGaIn (gallium indium eutectic alloy) is a metal material that has a room temperature melting point. Mostly, gallium and indium elements are exploited in the semiconductor industry, 
such as chips and LEDs. Recent research revealed that liquid metal has many interesting characteristics, such as swimming in electrolytes by interior or exterior stimuli.. ${ }^{2,27}$ Compared to mercury, EGaIn has a much lower vapor pressure and toxicity, which benefit its potential applications in biomedical areas. In this work, 76\% Ga, 14\% In EGaIn was exclusively implemented to form the worm robot.

\section{Experimental set up}

Channels and reservoirs with several different sizes and depths were implemented in our experiments. Three straight channels with widths of $6 \mathrm{~mm}, 8 \mathrm{~mm}, 10 \mathrm{~mm}$, a length of $150 \mathrm{~mm}$ and a depth of $6 \mathrm{~mm}$ were CNC machined on a PMMA (polymethylmethacrylate) transparent plate. These straight channels were utilized to verify the decisive factors of the dual-directional locomotion demonstrated by the worm. Another CNC machined PMMA pool was used to display how the head and tail of the worm performed differently when linked by a $0.5 \mathrm{~mm}$ thin copper rod. The size of the pool was $25 \mathrm{~mm} \times 100 \mathrm{~mm}$, with a depth of $8 \mathrm{~mm}$.

Several curved channels with angles of $30^{\circ}, 60^{\circ}, 90^{\circ}, 120^{\circ}$ and $150^{\circ}$ are FDM (fused deposition modeling) 3D printed. The width of the channels is $8 \mathrm{~mm}$, while the depth is $6.5 \mathrm{~mm}$. The printer is a Dobot Magician robotic arm with a precision of 0.1 $\mathrm{mm}$.

The loop channel and obstructive channels were SLA (stereo-lithography) 3D printed because of their irregular shapes. One of the obstructive channels had a $2 \mathrm{~mm}$ wide slit at the middle, while the other obstructive channel had a convergent and divergent joint section that connected the two reservoirs. All the obstructive channels had a width of 11 $\mathrm{mm}$ and a length of $58 \mathrm{~mm}$. The loop channel was a $4 \mathrm{~mm}$ deep rectangular groove with rounded corners. The width of the rectangle was $20 \mathrm{~mm}$, and the length was $75 \mathrm{~mm}$. A deeper reservoir was designed in the loop to store the liquid metal bulk and solution.

The electrolyte was a $0.5 \mathrm{~mol} \mathrm{~L}^{-1} \mathrm{NaOH}$ alkaline solution that filled all the channels and reservoirs aforementioned. Then, different volumes of liquid metal materials were injected into the channels or reservoirs within the different experiments. Both the solution and liquid metal can be drawn out with a syringe.

The images and ESI Movies $\uparrow$ were taken by a Huawei honor V8 smartphone dual camera module with a resolution of $1920 \times$ 1080. The zoom-in view in Fig. 5 was captured by a Supereye B011 USB digital microscope $(500 \times)$.

\section{Activation of peristalsis}

After set up preparation, the electrodes were used to apply a consistent voltage stimuli. Copper wires were implemented as electrodes that were connected to a constant voltage source (ZhaoXin KXN3030D). The range of the applied voltage was from $7 \mathrm{~V}$ to $23 \mathrm{~V}$. The tips of the electrodes were placed at opposite extremities of the grooves and dipped in alkaline solutions. After the voltage implementation, the locomotion of the worm initiated.

\section{Results and discussion}

In previous studies, the electric field would drive a sphere liquid metal droplet towards the anode electrode in alkaline or acid solution. ${ }^{26,28}$ The locomotion speed is relative to the voltage amplitude, which means droplets move faster under a higher voltage. The appropriate interpretation of the phenomenon is that the driving force is induced by the surface tension gradient, while the gradient is induced by the electric field implementation and electric double layer (EDL). The liquid metal on the droplet skin flows from a low surface tension area to a high surface tension area according to the Marangoni effect, which means it flows from the cathodic pole to the anodic pole. When the driving force overcomes the retardation force, the liquid metal droplet is propelled to move towards the anodic electrode.

Recently, our research found that liquid metal can also be controlled to move toward the cathodic electrode using an exterior electric potential. The essential factor of the phenomenon differing from previous research is the shape of the liquid metal droplet. When a droplet of liquid metal is injected into a thin long channel, the liquid metal forms a cigar or a worm shape rather than a round sphere in the free space due to the confinement of the channel. After voltage implementation, the liquid metal moves towards the cathode with a much slower speed compared to moving towards the anode. As the liquid metal bulk is slender, smooth and moving slowly, to some extent, it resembles an earthworm crawling on soil. So, we named it a "liquid metal worm," fitting its locomotive pattern. To clarify the relationship between the shape of the liquid metal worm and the direction of the locomotion, channels with different widths of $6 \mathrm{~mm}, 8 \mathrm{~mm}$ and $10 \mathrm{~mm}$ were implemented. Firstly, a bulk of $0.4 \mathrm{~mL}$ EGaIn alloy was injected into the $10 \mathrm{~mm}$ open top channel along with the $0.5 \mathrm{~mol} \mathrm{~L}^{-1} \mathrm{NaOH}$ solution. The channel was wide enough to contain the liquid metal without squeezing it. Thus, the liquid metal bulk was rounded like a ball. Two copper electrodes were placed at the two extremities of the channel and connected to the constant voltage power supply. Adjusting the output of the power source to $13 \mathrm{~V}$, we found that the liquid metal ball would dash towards the anode at a speed of $30 \mathrm{~mm} \mathrm{~s}^{-1}$, approximately. As is shown in Fig. 1(b), the blue graph illustrates the locomotion, while the navy top and right axis represent the coordinate of the blue line. Then, we switched the liquid metal ball into another parallel channel with a width of $6 \mathrm{~mm}$. Due to the constrain of the channel wall, the sphere would form into a much longer and more slender shape, which made it look like a worm. We filled the channel with $0.5 \mathrm{~mol} \mathrm{~L}^{-1} \mathrm{NaOH}$ solution and applied a $13 \mathrm{~V}$ voltage on both sides of the channel, which was a set up consistent with the previous one. Then, the worm would start to crawl forward towards the cathode copper wire electrode, but the speed of crawling was much slower, about $5 \mathrm{~mm} \mathrm{~s}^{-1}$ (Fig. 1(a)).

A supplementary experiment with a channel $8 \mathrm{~mm}$ in width was conducted, and the liquid metal worm also moved towards the cathode at a speed of $\sim 4.6 \mathrm{~mm} \mathrm{~s}^{-1}$. For all three 


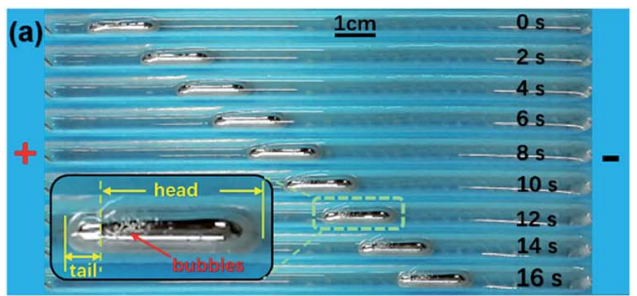

(c)

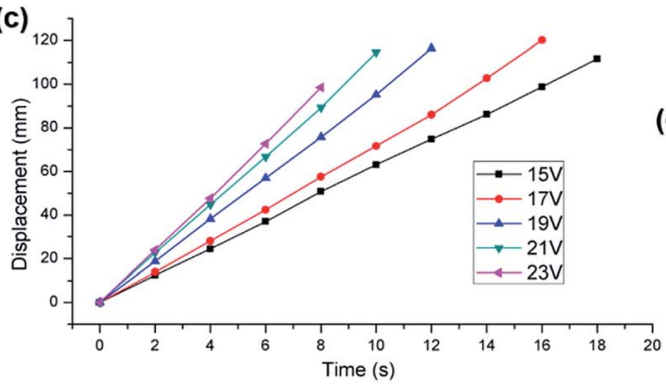

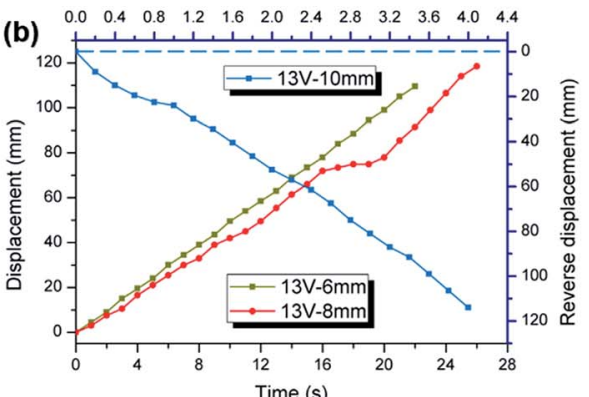

(d)

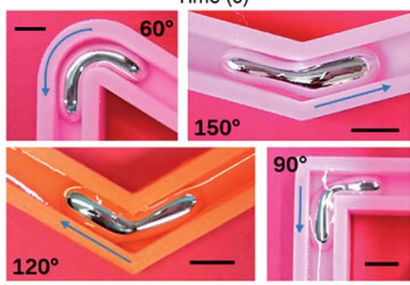

Fig. 1 Crawling locomotion of the liquid metal worm. (a) Liquid metal worm crawling towards the cathode. (b) Displacement diagram of the liquid metal movement in the channels with different widths. (c) Displacement diagram of the liquid metal locomotion under incremental voltages. (d) Liquid metal worms crossing corners with different angles. The blue arrows represent the motion directions. The scale bars are $1 \mathrm{~cm}$.

experiments, their displacement-time diagrams are plotted in Fig. 1(b), in which the blue descending line represents the liquid metal ball moving toward the anode in the $10 \mathrm{~mm}$ channel, while the red and brown ascending lines represent the liquid metal worm moving toward the cathode in the $6 \mathrm{~mm}$ and $8 \mathrm{~mm}$ channels, separately (ESI Movie S1†).

Further, the relationship between the magnitude of the applied voltage and locomotive velocity was also unveiled by a series of experiments. With the same environmental parameters as the previous experiments $\left(6 \mathrm{~mm}\right.$ channel and $0.5 \mathrm{~mol} \mathrm{~L}^{-1}$ $\mathrm{NaOH}$ solution), the magnitude of the voltage was varied to examine the relevance. The voltages start from $15 \mathrm{~V}$ and ascend to $23 \mathrm{~V}$ in intervals of two volts. The volume of liquid metal is 0.4 $\mathrm{mL}$. We can illustrate the displacement-time graphs utilizing the experimental data, and we noticed that with a higher applied voltage, the liquid metal worm would crawl faster toward the cathodic electrode, as shown in Fig. 1(c) (also shown in ESI Movie $\mathrm{S} 2 \dagger)$. The close up of the snapshot of the liquid metal droplet in motion shows that there is an obvious separation between the two sides of the liquid metal worm. The head of the worm is bigger and longer than its rear part, which has a smaller diameter along with a shorter length. Also, some tiny bubbles attached to the joint section between the worm head and tail. Bubbles floating in the $\mathrm{NaOH}$ solution can be regarded as indicators of the vortex flow, which shows that the head and the tail of the liquid metal worm have different locomotive tendencies towards opposite directions within the open top channel. It is more likely that the two ends of the liquid metal worm are competing with each other to drag the whole body in its own direction. Nevertheless, the worm was driven towards the cathode in this condition, which means that the head of the liquid metal worm has a higher dragging force compared with its counterpart.

The liquid metal worm was also tested in several curved channels with angles of $30^{\circ}, 60^{\circ}, 90^{\circ}, 120^{\circ}$ and $150^{\circ}$. The volume of the metal is about $1 \mathrm{~mL}$, and the applied voltage is $15 \mathrm{~V}$
(Fig. 1(d)). The results showed that the worm could cross all the corners but $30^{\circ}$, where the worm was cut in half by the sharp corner. The times for passing through the corner are $5.2 \mathrm{~s}, 4.4 \mathrm{~s}$, $7.8 \mathrm{~s}$ and $5.4 \mathrm{~s}$ with angles of $60^{\circ}, 90^{\circ}, 120^{\circ}$ and $150^{\circ}$, respectively. The shape of the worm also implies that the worm head and tail may have different motion characteristics.

To prove our conjecture, we increased the voltage to a relatively higher value of $19 \mathrm{~V}$ in the straight channel. The head and the tail of the worm moved in different directions, and the middle body was stretched into a long slim waist. At this time, the shape of the worm was more like a dumbbell, shown in Fig. 2(a). If the exterior voltage continued for a second, the joint of the two ends broke, and the body of the worm was torn into two individual parts that moved in opposite directions. At the same time, the slim waist shifted onto the longer part (ESI Movie S3 $\uparrow$ ). This phenomenon is quite similar to the regeneration of the planarian flatworm, which belongs to a separate phylum from earthworms. When a planarian flatworm is cut into several segments, every piece is able to reform the entire body. In our experiments, the separate parts of the liquid metal worm did not grow larger, but they maintained functionality and could be driven by an exterior voltage to crawl.

The volume of the liquid metal was decreased to $0.2 \mathrm{~mL}$ in the open top channel with a width of $6 \mathrm{~mm}$. Thus, the longitudinal length of the droplet was shorter, which makes the shape more round. The channel was filled with an alkaline solution, and after a $23 \mathrm{~V}$ voltage implementation, the shorter and rounder liquid metal worm oscillated forward and backward in the channel. In this specific condition, the driving force towards the anode and cathode is almost equivalent, which makes the worm stay in an unstable state. However, the tugging force is not strong enough to tear the worm apart. In other words, this state is a turning point between the cathode oriented and anode oriented locomotion, and it further supported our former observation. 


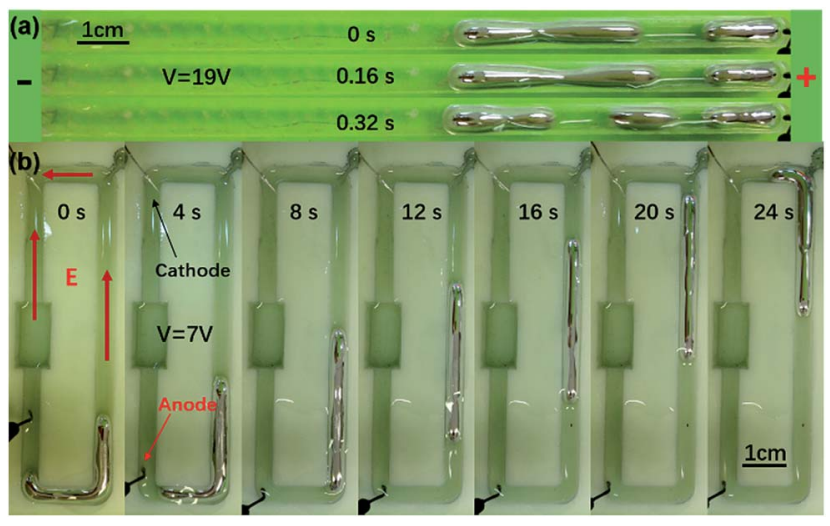

Fig. 2 Splitting and deflected crawling of the liquid metal worm. (a) The liquid metal worm was torn apart under a $19 \mathrm{~V}$ applied voltage. (b) The worm wriggled in a loop open-top channel with an applied voltage of $7 \mathrm{~V}$. Red arrows represent electric field directions in the electrolyte.

We also placed the worm in a SLA 3D printed loop channel to inspect whether it can steer along the $4 \mathrm{~mm}$ wide ditch as a real worm. One millilitre of the EGaIn alloy was injected into the open top channel along with the $\mathrm{NaOH}$ solution. Two copper electrodes were submerged into the solution at neighbouring corners, as is shown in Fig. 2(b), and the output of the electrodes was reduced to 7 volts to prevent splitting. When the power was switch on, the liquid metal worm gradually accelerated and wriggled along the channel. The entire length of the worm, which was about $35 \mathrm{~mm}$, was much longer than the short side of the channel, thus, the worm head needed to swirl its direction at the corners, as well as other parts of the body (also shown in ESI Movie S4 $\dagger$ ). With a closer observation, it was found that the worm shivers as it crawled forward, and the solution in proximity was stirred, generating a small vortex and ripples. It takes about 24 seconds to travel half the entire loop. Fig. 2(b) displays the snapshots of the locomotion process every four seconds. The upper surface of the liquid metal worm is not completely covered by the aqueous solution, but it seems that the movement is not evidently affected.

Considering that this metal worm is composed of liquid material, intuitively, we have a strong interest in whether it can deform freely in twisted spatial conditions. Such an attribute would be very attractive and pragmatic in many fields, especially in robotics and biomedical engineering. For instance, if a vascular robot can narrow through a vascular valve and return to its original shape and function, then the region of the movement could be extended and more work could be accomplished, such as drug delivery or thrombus removal.

To inspect whether the worm could move across a slit or a thin channel, we designed two open top channels with narrow joints in the midst, as shown in the right side of Fig. 3(a) and (b). The bright green represents 3D printed channel walls, while the pale yellow represents the bottom of the channel. The cathode electrode is placed at the upper end, and the anode at the lower extremity. There are fence structures at the two ends of the channel to isolate the electrodes because the electrolysis generated bubbles may diffuse among the solution surface and disturb our observation. The liquid metal worm is set in the lower reservoir initially. Because the worm is more stout, a higher voltage was needed, and we set the power source output to $17 \mathrm{~V}$ to drive the worm.

The first channel has a gradually contracted middle section, which is formed by two arcs, and the narrowest position has a width of $3 \mathrm{~mm}$. The convergent curve would help the worm to deform without creating too much retardation force. When the power is on, the cigar shape liquid metal worm started staggering to the neck, which looks like a de Laval nozzle. The head of the worm would squeeze to an extrusive point and distort to adapt the shape of the neck (ESI Movie S5†). In less than $0.2 \mathrm{~s}$,
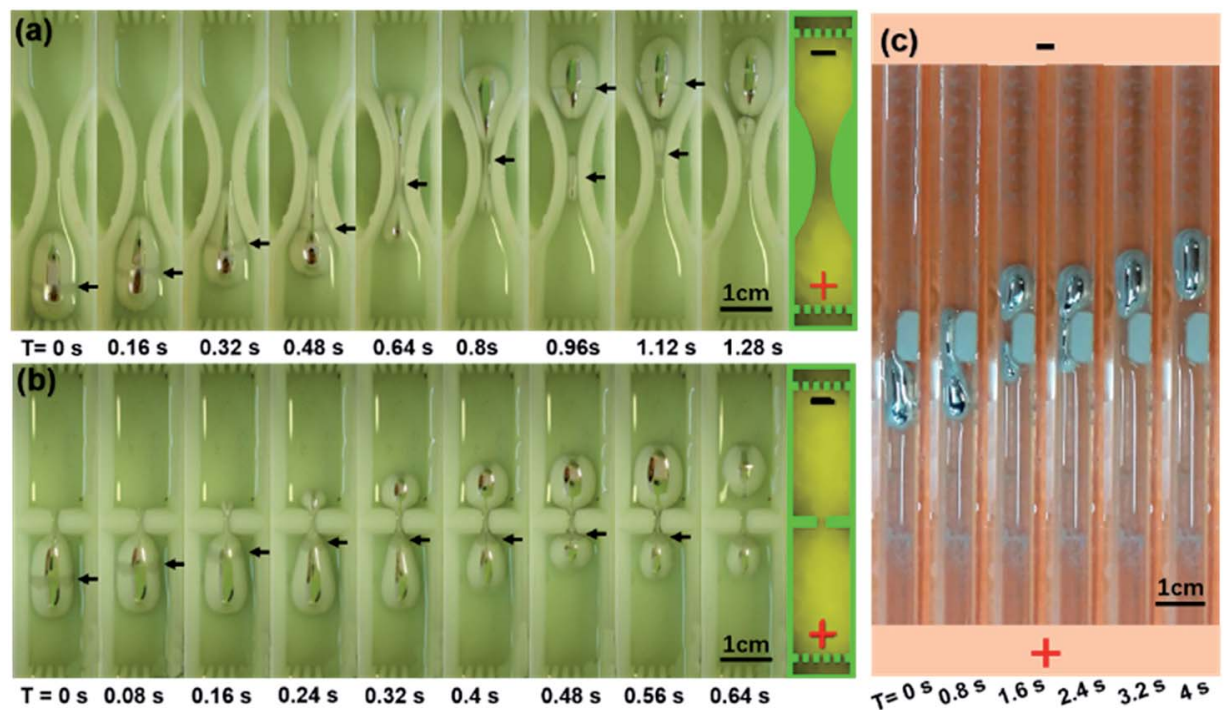

Fig. 3 Liquid metal worm crossing different types of obtrusive channels. (a) The worm crossed a contractile-expanded channel. (b) The worm crossed a $2 \mathrm{~mm}$ wide slit. (c) The worm bypassed an asymmetric obstacle within a straight channel. 
the front of the worm crossed the neck, and the tail shrinks to a tiny long droplet. Sometimes the tail may break apart from the worm, as is shown in the seventh snapshot. During the crossing, a ring of dim grey stripe appeared at the waist of the worm, which is labelled by the short black arrows, and it also moved along the body and stops at the narrowest position. It can also be seen that when the worm tail departs from the body, the break point is right at the stripe. In the end, both the head and the tail of the liquid metal worm run across the contractile channel and recover their original morphology.

To further test the crossing capability of the worm, a more difficult obstructive channel was designed to evaluate its deformability. Two reservoirs at the upper and lower parts were connected by a small slit (Fig. 3(b)), while the width and the length of the channel were consistent, $11 \mathrm{~mm}$ and $58 \mathrm{~mm}$, respectively. In our second experiment, after the voltage activation, the worm would wriggle and move towards the cathode electrode as well as the gap. At the time when the worm contacts the middle wall, a tiny bud generated and strode into the slit. The bud grew longer inside the gap as the worm moved forward. When the bud bumped out of the gap, it grew to a sphere pattern and increased its volume (ESI Movie S6†). At the same time, the grey stripe at the waist of the worm would move towards the slit and be stuck at the barrier, which can be seen from the first four snapshots. The size of the worm reaching into the upper channel keeps increasing while the lower part keeps decreasing until more than half of the worm transferred into the upper reservoir. When the two liquid metal parts are connected by a thin liquid metal line, this connection might break by the disturbance of peristalsis, shown in the last snapshot. Thus, the worm would split with the head and tail staying in the upper and lower channel, respectively.

Both experiments above were implemented with symmetrical obtrusive gaps. Another experiment with an asymmetrical gap was also conducted in the $8 \mathrm{~mm}$ width straight channel. A rubber cube with a $4 \mathrm{~mm}$ thickness and $10 \mathrm{~mm}$ length was adhered to one side of the PMMA channel wall. Thus, an asymmetrical barrier was formed. When a $13 \mathrm{~V}$ voltage was applied at the two extremities, the worm with a volume of 0.5 $\mathrm{mL}$ deformed to bypass the rubber cube, as is shown in Fig. 3(c) and ESI Movie S7.†

We used an HD camera to better observe the dim grey stripe on the waist of the worm, which was placed in a small reservoir, as is shown in Fig. 4(b) and (c). The edge shapes of the stripes were different at the two sides, which are labelled by the cyan dashed line. The border close to the cathode was a straight line, while the edge close to the anode was a crescent curved line. At the two extremities of the bulk droplet that were not covered by a thin stripe, the flow at the liquid metal surface and adjacent solution vortex can be observed by the floating of the generated bubbles.

When a liquid metal worm was placed within the absence of an external electric field, an asymmetric reactivity would be generated on the surface of the conductive liquid metal, according to bipolar electrochemistry. ${ }^{29}$ Setting $E_{\mathrm{a}}$ and $E_{\mathrm{c}}$ as the potential of the anode and the cathode, $D$ as the distance between the two electrodes, the electric field value $\delta$ is given by

$$
\delta=\frac{E_{\mathrm{a}}-E_{\mathrm{c}}}{D}
$$

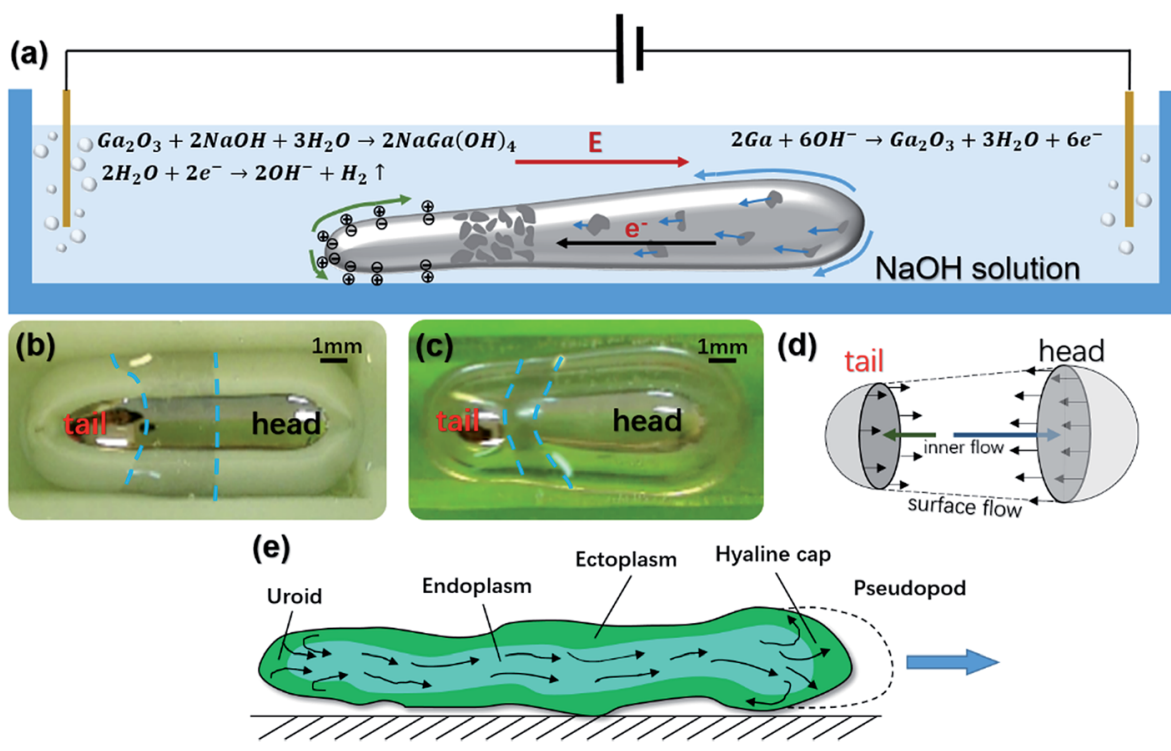

Fig. 4 Schematic diagram of the liquid metal worm locomotion and the way for an analogous amoeba. (a) Schematic diagram of the liquid metal worm locomotion. Green and blue curved arrows represent the surface flow among the worm skin. Red straight arrow represents the direction of the electric field while the black straight arrow represents the migration of electrons within the body. Deep grey fragments represent gallium oxide generated upon the skin. (b) and (c) Zoomed in view of the liquid metal worm and its dim stripe that separates the head and the tail. (d) Flow directions of the bulk liquid metal corresponding to (b) and (c). (e) Schematic diagram of the amoeba locomotive pattern. Endoplasm transformed to ectoplasm at the hyaline cap and restored at uroid. Pseudopod is elongated by the endoplasm flow. 
and the imposed potential drops linearly across the solution in a first order approximation. The polarization potential difference that occurs between the two extremities of the worm can be calculated as:

$$
\Delta V=\delta l
$$

with $l$ being the length of the conducing worm. This value is related to the reactivity at the polarized interface, which is

$$
2 \mathrm{Ga}+6 \mathrm{OH}^{-} \rightarrow \mathrm{Ga}_{2} \mathrm{O}_{3}+3 \mathrm{H}_{2} \mathrm{O}+6 \mathrm{e}^{-}
$$

and

$$
\mathrm{Ga}_{2} \mathrm{O}_{3}+2 \mathrm{NaOH}+3 \mathrm{H}_{2} \mathrm{O} \rightarrow 2 \mathrm{NaGa}(\mathrm{OH})_{4}
$$

The extremity closer to the cathode, which is the anodic pole, would impose the first reaction. While along the worm skin, the generated oxide layer would be consumed by the second reaction. ${ }^{30}$ When a bulk of liquid metal is confined in a slim channel, the characteristic length $l$ would increase, thereby raising the $\Delta V$, which consolidates the polarization effect.

Inside the liquid metal worm, electrons flow from the anodic pole to the cathodic pole. With the formation of a solid-state oxide $\mathrm{Ga}_{2} \mathrm{O}_{3}$ thin layer, the distal anodic pole would have the lowest surface tension. According to the Marangoni effect, the liquid would flow from the low surface tension area to the high surface tension area. Because there is less oxide formation away from the distal anodic pole, the skin of the liquid metal worm would flow from the anodic pole towards the waist of the worm (Fig. 4(d)).

The solid state oxide $\mathrm{Ga}_{2} \mathrm{O}_{3}$ would float among the skin and be carried to the middle section of the worm by the surface fluxion. With the accumulation of the oxide, a dim stripe would generate at the waist. Against the flow from the anodic pole to the middle position, the stripe would face a reverse fluxion, which stops its advance towards the cathodic pole. When approaching the posterior part of the worm, the oxide would reduce to the liquid state gallium by migrated electrons. Thus, the amount of gallium oxide reaches a dynamic balance. $\mathrm{NaOH}$ also contributes to the consumption of gallium oxide, which slightly dissolves in alkaline solution. ${ }^{31,32}$

When the worm skin moves, it propels the solution at the vicinity as well as the interior channel surface. The propulsion would give the worm a reaction force to drive it moving forward. This locomotion pattern analogizes the movement of an amoeba, which has a convertible exterior ectoplasm and interior endoplasm. Endoplasm converts to ectoplasm at the hyaline cap and ectoplasm transforms back to the endoplasm at the uroid. Inside, the endoplasm flows from the tail to the cap and elongates the pseudopod. With ectoplasm adhesively fixed with the plate beneath the amoeba, an amoeboid movement could be achieved (Fig. 4(e)). Here, we could regard the oxide of gallium as the "ectoplasm" and the liquid state alloy as the "endoplasm", and these two movements are quite similar to each other.

This mechanism can also be utilized to explain the capability of narrowing through a contractile channel. As the anodic pole at the worm head contacted with the convergent channel wall, the surface tension lowered by the oxide would allow the head to deform to the confinement of the channel. With the existence of the surface tension gradient, the skin movement would drive the tip point of the head to bump out the slit, just like the pseudopod of an amoeba. Inside the body, liquid metal flows from the waist to the head to enlarge the bulge. When crossing the obstacle, the anterior body would pull the other part out through the slit. The movement in the loop channel suits the same interpretation as well.

The gallium oxide that accumulated at the waist limited the mobility of the skin, thus, when the bulk inside the liquid metal crossed the barrier, the dim stripe would stay in front of the gap, as is shown in Fig. 3(a) and (b). As the tail shrinks, the waist of the worm also contracted to a thin line since the tail and the head have different kinetic trends. In most cases, the worm would break when the waist got too thin to bridge the two parts. If the worm is smaller and the gap is a little wider, the worm could cross the gap without any interruption.

While if we look at the other side of the worm, the tail skin also flows but in an opposite direction. When the liquid metal is immersed in the $\mathrm{NaOH}$ alkaline solution, an electric double layer is generated on the interface, in which the liquid metal side is negatively charge while the solution is positively charged. The phenomenon could be interpreted by the Lippmann's equation:

$$
\gamma=\gamma_{0}-\frac{1}{2} c V^{2}
$$

which is also mention in previous work. ${ }^{27} \gamma$ is the surface tension, $c$ represents the capacitance of the electric double layer (per unit area), $\gamma_{0}$ represents the maximum surface tension when the potential difference across the EDL $V$ is zero. After implementing an exterior electric field, the outermost point at the cathodic pole would have a higher electric charge density, so the potential difference $V$ would increase. According to Lippmann's equation, the surface tension $\gamma$ would decrease at this position. Considering the Marangoni effect, the skin would flow from the tip point to the middle joint of the worm, which would generate a resistance to drag the worm backward. Because the tail has an opposite locomotive tendency, the worm would crawl quite slowly compared to liquid metal locomotion in previous research. $^{26}$

If this opposite locomotive tendency is strong enough, the worm might be torn apart at the joint section, as is shown in our aforementioned experiment. After which, the initial tail would rush to the anode electrode while a new tail formed at the end of the head. Or, if the worm is too stout to split, the dragging resistance equals the pulling force, and the worm may oscillate in the channel with the disturbance of the surface roughness and vortex.

To prove our explanation, we conducted another experiment in which two liquid metal droplets with the same volume of 0.15 $\mathrm{mL}$ were connected by a fixed copper rod with a diameter of 0.5 $\mathrm{mm}$. Both the rod and two droplets were immersed in a $0.5 \mathrm{~mol}$ $\mathrm{L}^{-1} \mathrm{NaOH}$ solution contained within a $25 \mathrm{~mm} \times 100 \mathrm{~mm}$ transparent pool, shown in Fig. 5(a). The copper rod had a solid 

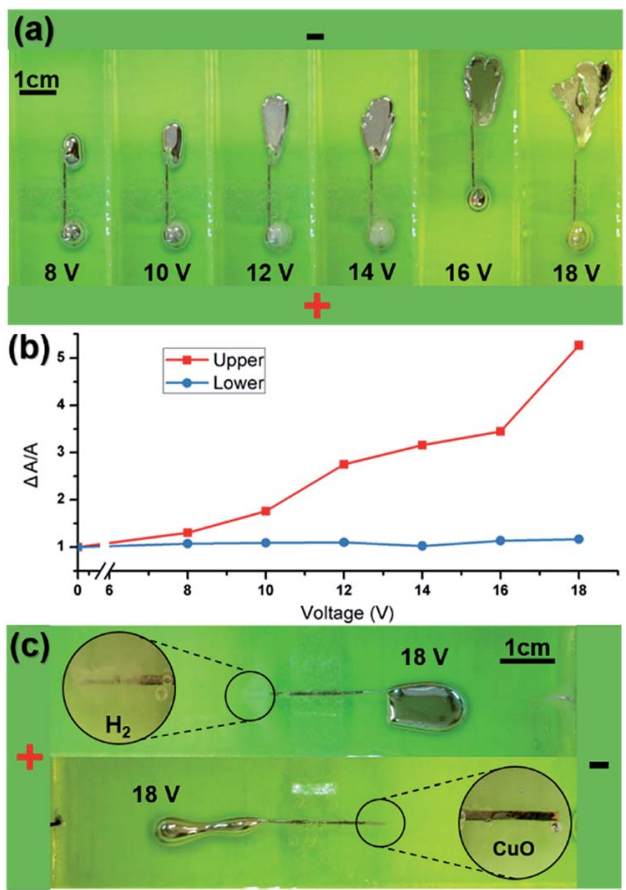

Fig. 5 Liquid metal droplet connected by a copper rod. (a) The droplet closer to the cathode (upper) would expand under different voltages, while the other one (lower) would generate gases. (b) Area changes of the two droplets under incremental voltages. (c) The tip of the copper rod would generate $\mathrm{H}_{2}$ bubbles (top) or be oxidized to black $\mathrm{CuO}$ (bottom) according to the positions of the electrodes.

surface, thus, liquid metal cannot flow from one droplet to the other. We applied incremental voltages to the solution, starting from $8 \mathrm{~V}$ to $18 \mathrm{~V}$ at intervals of 2 volts. The droplet closer to the anode would flatten while its counterpart would struggle to get rid of the rod connection (ESI Movie S8 $\dagger$ ). The flattened deformation at the upper droplet is highly analogic to the experiment that the anode contacted with the liquid metal droplet. ${ }^{33}$ As we elucidated before, this is caused by accumulation of gallium oxide. Without any reduction or surface transportation, the oxide would stay where it is and can only be consumed by the $\mathrm{NaOH}$ chemical reaction, which has a speed far below the oxide generation. At the very beginning the surface tension gradient would still exist and makes the flattened puddle expand outward. With the surface area increasing and the oxide covering the entire surface, the oxidation would balance with the consumption process. In the end, the puddle would stop expansion and become steady. The thick oxide layer had limited the mobility of liquid metal.

The other droplet is supplied with electrons conducted by the copper rod, so the oxide cannot exist and $\mathrm{H}_{2}$ bubbles are generated because of the reduction. At this end, the EDL is stable as well as the surface tension gradient. The droplet shook to depart from the rod and was adhered by metal wetting.

When the external voltage increased, the oxide layer upon the upper droplet would be thicker and caused a more deplanate shape. Here, we used the relatively occupied area $\left(\frac{\Delta A}{A}\right)$ as an indicator of how much the shape had changed. In Fig. 5(b), we can see that the upper droplet occupied area increased about five times compared to its original state (red line), while the area change of the lower droplet is negligible. We removed one droplet and observed the copper end with a digital microscope, and we could see that the copper tip was oxidized to black $\mathrm{CuO}$ or generated massive gas when the tip was close to the cathode or anode, respectively.

In another experiment, a liquid metal worm crawled forward in a straight channel, and when it contacted the cathode wire electrode, it bounced back instantaneously with a high speed and was restored to its original state in $\sim 0.5$ second. This "crawling forward-contact-bounce back" process would repeat periodically as long as the voltage was implemented (also shown in ESI Movie S9†). Several cycles were recorded by the displacement-time diagram (Fig. 6), from which we can calculated that the period is about $2.5 \mathrm{~s}$. The right-side figures are snapshots of one period with a time interval of $0.4 \mathrm{~s}$. The red and the black dashed lines represent the anode and cathode, respectively.

This phenomenon can also be interpreted by the formation and reduction of the gallium oxide layer. When the worm contacted the cathode, the oxide layer is reduced and removed to expose the inner EGaIn alloy. Thus, the only surface tension
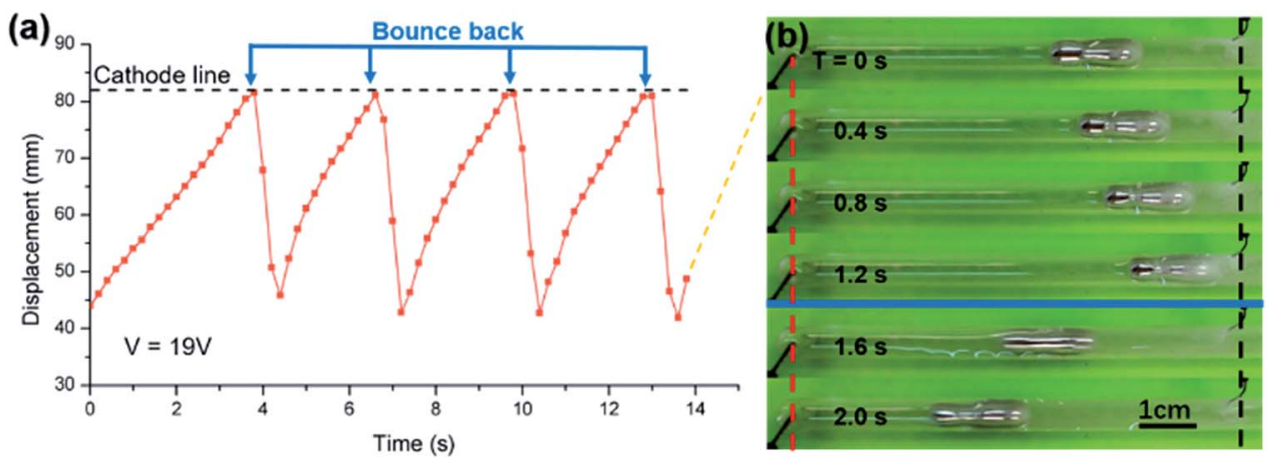

Fig. 6 Bouncing back locomotion of the liquid metal worm. (a) Displacement of the locomotion. The yellow dashed line represents the continuous periods. (b) Snapshots of the locomotion. The red and black dashed line represent the anode and cathode respectively. The worm moved rightward above the blue line but leftward below the line. Applied voltage is $19 \mathrm{~V}$. 
gradient is caused by the potential difference across the EDL, which makes the worm rush back and separate with the cathode. Afterward, the oxide layer was regenerated by the polarization effect, and the worm would crawl back in the original direction. Both the velocity and the periodicity are embodied in the graph, where the dashed black line marked the position of the cathode.

\section{Conclusions}

In summary, we have unveiled a novel locomotive characteristic of EGaIn liquid metal, which is dubbed "worm-like locomotion." The speed of the locomotion is much lower compared with the anode oriented movement that we had discovered before. The reason for the low speed is endowed by the surface oxide layer generation and Janus locomotion. The oxide layer also reduced the surface tension significantly, and the liquid metal worm can squeeze and narrow across a highly confined gap. This characteristic endows the liquid metal worm with the capability to overcome varied obstacles such as a vascular flap or thrombus. The detailed interpretation is introduced and strengthened by several experiments that proved the formation and reduction of the oxide layer as well as the retardation force caused by worm tail.

Such a liquid metal worm can be utilized in many pragmatic fields such as microfluidics, miniaturized vehicle, flexible micro-bot and so on. If we attach other solid structures or modified pharmaceutical molecules, then the worm could be regarded as a functional transporter or delivery system. The velocity can be controlled by an external or mounted voltage supply while the size of the worm could be adjusted according to the specific application. We hope that this research could inspire other researchers in related areas and advance the development of smart, biomimetic and flexible robots in the coming future.

\section{Acknowledgements}

This work is partially supported by the Dean's Research Funding and Frontier Project of the Chinese Academy of Sciences.

\section{References}

1 P. Arena, C. Bonomo, L. Fortuna, M. Frasca and S. Graziani, IEEE Trans. Syst. Man Cybern. B Cybern., 2006, 36, 1044-1052.

2 C. D. Onal, R. J. Wood and D. Rus, IEEE ASME Trans. Mechatron., 2013, 18, 430-438.

3 E. Steltz, A. Mozeika, N. Rodenberg, E. Brown and H. M. Jaeger, IEEE/RSJ International Conference on Intelligent Robots and Systems, 2009, pp. 5672-5677.

4 A. S. Boxerbaum, K. M. Shaw, H. J. Chiel and R. D. Quinn, Int. J. Robot. Res., 2012, 31, 302-318.

5 C. Huang, J. A. Lv, X. Tian, Y. Wang, Y. Yu and J. Liu, Sci. Rep., 2015, 5, 1-8.

6 Y. Yao and J. Liu, RSC Adv., 2016, 6, 56482-56488.
7 D. Rus and M. T. Tolley, Nature, 2015, 521, 467-475.

8 W. Wang, W. Duan, S. Ahmed, T. E. Mallouk and A. Sen, Nano Today, 2013, 8, 531-554.

9 J. Roche, S. Carrara, J. Sanchez, J. Lannelongue, G. Loget, L. Bouffier, P. Fischer and A. Kuhn, Sci. Rep., 2014, 4, 6705.

10 R. F. Shepherd, F. Ilievski, W. Choi, S. A. Morin, A. A. Stokes, A. D. Mazzeo, X. Chen, M. Wang and G. M. Whitesides, Proc. Natl. Acad. Sci. U. S. A., 2011, 108, 20400-20403.

11 S. A. Morin, R. F. Shepherd, S. W. Kwok, A. A. Stokes, A. Nemiroski and G. M. Whitesides, Science, 2012, 337, 828-832.

12 A. A. Solovev, S. Sanchez, M. Pumera, Y. F. Mei and O. G. Schmidt, Adv. Funct. Mater., 2010, 20, 2430-2435.

13 H. Philamore, J. Rossiter, A. Stinchcombe and I. Ieropoulos, in Intelligent Robots and Systems (IROS), IEEE/RSJ International Conference, 2015, pp. 3888-3893.

14 A. A. Stokes, R. F. Shepherd, S. A. Morin, F. Ilievski and G. M. Whitesides, Soft Robotics, 2014, 1, 70-74.

15 Q. Wang, Y. Yu, K. Pan and J. Liu, IEEE Trans. Biomed. Eng., 2014, 61, 2161-2166.

16 B. C. Jayne, Copeia, 1986, 915-927.

17 B. C. Jayne, J. Morphol., 1988, 197, 159-181.

18 D. Zarrouk, I. Sharf and M. Shoham, Robotics and Automation (ICRA), IEEE International Conference, 2010, pp. 1574-1579.

19 T. Nakamura, T. Kato, T. Iwanaga and Y. Muranaka, J. Robot. Mechatron., 2006, 18, 299.

20 A. M. Bertetto and M. Ruggiu, Advanced Intelligent Mechatronics, Proceedings, IEEE/ASME International Conference, 2001, vol. 2, pp. 1226-1231.

21 M. E. Ingram, Doctoral dissertation, Virginia Polytechnic Institute and State University, 2006.

22 Y. Shirai, K. Minami, W. Nakanishi, Y. Yonamine, C. Joachim and K. Ariga, Jpn. J. Appl. Phys., 2016, 55, 1102 A2.

23 J. Li, I. Rozen and J. Wang, ACS Nano, 2016, 10, 56195634.

24 M. Srisa-Art and Y. Furutani, Bull. Chem. Soc. Jpn., 2015, 89, 195-202.

25 J. Zhang, S. Yan, D. Yuan, G. Alici, N. T. Nguyen, M. E. Warkiani and W. Li, Lab Chip, 2016, 16, 10-34.

26 L. Sheng, J. Zhang and J. Liu, Adv. Mater., 2014, 26, 60366042.

27 J. Zhang, Y. Yao, L. Sheng and J. Liu, Adv. Mater., 2015, 27, 2648-2655.

28 L. Hu, L. Wang, Y. Ding, S. Zhan and J. Liu, Adv. Mater., 2016, 28, 9210-9217.

29 G. Loget, D. Zigah, L. Bouffier, N. Sojic and A. Kuhn, Acc. Chem. Res., 2013, 46, 2513-2523.

30 R. C. Gough, J. H. Dang, M. R. Moorefield, G. B. Zhang, L. H. Hihara, W. A. Shiroma and A. T. Ohta, ACS Appl. Mater. Interfaces, 2015, 8, 6-10.

31 J. Zhang, Y. Yao and J. Liu, Science Bulletin, 2015, 60, 943951.

32 L. Sheng, Z. He, Y. Yao and J. Liu, Small, 2015, 11, 5253-5261. 33 J. Zhang, L. Sheng and J. Liu, Sci. Rep., 2014, 4, 7116. 